\title{
Research on the method of UG secondary development to the calculating plane dimension chain
}

\author{
Pang Lijun ${ }^{1, a}$, Liu Yongping ${ }^{2, b}$, Fu Rongrong ${ }^{3, \mathrm{c}}, \mathrm{Ji} \mathrm{Li}^{4, \mathrm{~d}}, \mathrm{Li}_{\text {Y Yan }}^{5, \mathrm{e}}$ \\ ${ }^{1,2,4}$ College of electromechanical engineering, Shenyang Aerospace University Shenyang, China \\ ${ }^{3}$ School of Mechanical Engineering\&Automation, Northeastern University, Shenyang, China \\ ${ }^{5}$ Qindao College, Qingdao Technological University, Qingdao, China \\ aplj7862@126.com , ${ }^{\mathrm{b}} 710622852 @ q q . c o m,{ }^{\text {c }}$ rong_fu1102@yahoo.com.cn, djilirory0211@gmail.com \\ ly_happyhappy@126.com
}

Keywords: plane dimension chain, projection method, UG secondary development, VB.NET

\begin{abstract}
In the analysis of the process of the complex plane dimension chain, if the influences of the component loops on the closed loops are nonlinear variation, the process of solution-finding will be complicated and tedious. Because we must apply mathematics principle to solve the closed loop and make a list of the dimension chain equation. In order to solve the above problems, on the basis of deeply researching the mathematical model of the dimension-chain-calculating method, we used projection method to analyze and extremum method to calculate the plane dimension chain, and finally finished the work by computer. Moreover, in the UG platform, we have completed operation interface employing VB.NET.
\end{abstract}

\section{Introduction}

In the mechanical design and manufacturing, the calculation problems of two dimensional plane dimension chain exist in all kinds of geometric construction such as accuracy analysis of plane mechanism, technology assembly, and tolerance conversion. Process designers usually calculate size chain by applied mathematics principle, establishing and solving dimension chain equations manually which are needed by them. The amount of the dimension chain loops is huge and the judgment of increasing and decreasing is difficult, which result in the errors of the calculation results of the unknown loops, and make the process complicated, tedious, workload, and consuming more time.

In order to solve the above problems and improve product digital management level, using computer aided in the designing process is a kind of effective method. We applied projection analysis method of plane dimension chain and extremum calculating method to develop UG NX aided analysis software of plane dimension chain calculation ${ }^{[1-3]}$. If the computing problem of dimensional chain is solved, it will make the process designer liberate from complicated job, and pay more attention to improve the quality of the products, the technology level.

\section{The method of plane dimension chain}

Plane dimension chain is the essential combination of linear dimension chain and angle dimension chain and each loop is arranged in one or several parallel planes according to angle relations. Linear dimension chain is a special case of the plane dimension chain. The auxiliaries dimension are brought in the plane dimension chain for solving a closed loop. It may need to draw one or more planes size chain charts. Therefore, the establishment and solving of the dimension chain equation are more complex than linear dimension chain solving.

Projection method for the calculation of plane dimension chain is applied in this paper. We used the projection method to solve the questions of the plane dimension chain through the projection relation decomposing for one dimensional (linear) dimension chain. Fig.1 shows the projection method, and the plane linear dimension chain relation equation is solved by the projection relation. And the result can be obtained by using the calculational method of the dimension chain. 

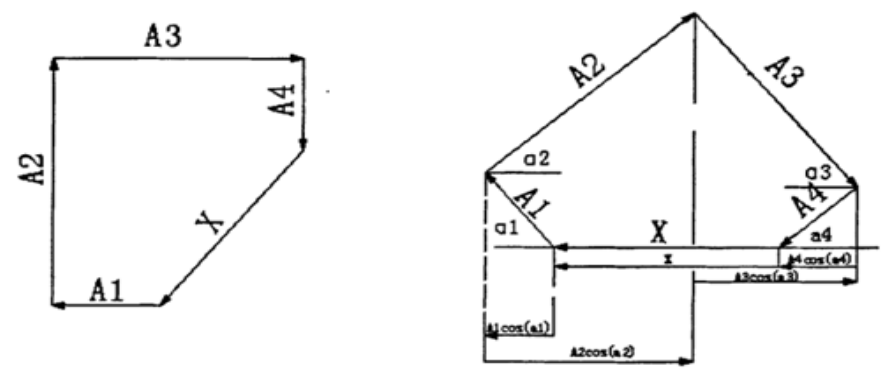

Fig.1 Projection method

\section{Calculation formula of plane dimension chain}

We projected the component loop to some directions, and found out the relational equations of the closed loop between all relevant component loops. The method for realizing the problem of two dimensional plane dimension chain is to transform it into a dimensional linear dimension chain. We builded a form of linear dimension chain to determine between the closed loop and the component loop relationship of the linear dimension chain. The calculation of linear dimension chain can be divided into the extremum method and the probability method. This paper only used extremum method. The equation of closed loop can be generally expressed as:

$$
A_{0}=f\left(A_{1}, A_{2}, \cdots, A_{i}\right)
$$

According to the concept of total differential, when the variable of each component loop produces small increment, the increase of the close loop is:

$$
d A_{0}=\sum_{i=1}^{n} \frac{\partial f}{\partial A i} d A_{i}
$$

Among them: $\frac{\partial f}{\partial A i}$ is transferring coefficient $\xi_{i}$, it could be positive or negative number. When $\frac{\partial f}{\partial A i}$ is positive number, $A_{i}$ is the increasing loop. When $\frac{\partial f}{\partial A i}$ is negative number, $A_{i}$ is the decreasing loop. If the upper deviation or lower deviation of each loop instead of the small incremental $d A_{i}$, we can approximately get upper and lower deviation of the closed loop $A_{0}$. The upper deviation $E S_{0}$ of the closed loop is:

$$
E S_{0}=\sum_{i=1}^{m}\left(\frac{\partial f}{\partial A_{i}}\right) E S_{i}+\sum_{j=1}^{n-m}\left(\frac{\partial f}{\partial A_{j}}\right) E I_{j}
$$

The lower deviation $E I_{0}$ of the closed loop is:

$$
E I_{0}=\sum_{i=1}^{m}\left(\frac{\partial f}{\partial A_{i}}\right) E I_{i}+\sum_{j=1}^{n-m}\left(\frac{\partial f}{\partial A_{j}}\right) E S_{j}
$$

Among them: $E S_{i}$ and $E I_{i}$ is the upper and lower deviation of the increasing loop, $E I_{j}$ and $E S_{j}$ is the upper and lower deviation of the decreasing loop.

\section{Angle prediction of plane dimension chain}

In the actual plane dimension chain diagram, not all length loops and projected directions angle are known, a lot of needed informations must be predicted through the other angle loop. In the prediction process, the main use of the dimension chain vector is the polygon angle between adjacent dimension loops. The calculating process is shown in Fig.2. Selecting A0 for projection edge $\alpha$ is an angle of A1 and A0.

We can regard A1 as counter clockwise in deflect $180^{\circ}-\alpha$ on the basis of A0, and get the angle 
of between $\mathrm{A} 1$ and projection edge is $\alpha_{1}=180^{\circ}-\alpha$, the angle between A1and A2 is $\beta$ we can regard A2 as counter clockwise in deflect $180^{\circ}-\beta$ on the basis of $\mathrm{A} 1$, and get the angle between A1 and projection edge is $\alpha_{2}=\alpha_{1}+180^{\circ}-\beta=180^{\circ}-\alpha+180^{\circ}-\beta$. The other side and the edge of the projection angle can be got by a similar way.

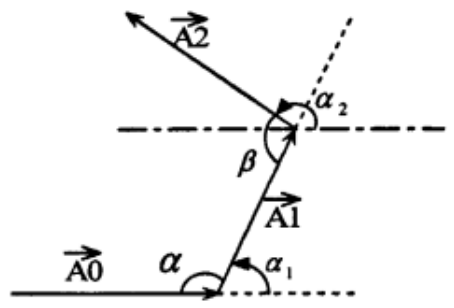

Fig.2 The method of vector angle prediction

\section{The calculation of UG secondary development plane dimension chain}

In order to ensure the correctness of dimensional chain calculation in the application of computer calculating plane dimension chain, some steps are needed to be followed. Firstly, we must draw the right dimension relations, and make sure the close loop and component loop according to the processing of this part or machine assembly process and the relationship among dimension. Secondly, in order to draw the right dimension chain chart, for the first, the intuitive three-dimensional parts must be conducted two-dimensional projection through the UG NX software function. Within each plane dimension project to the specified coordinates, which make the information of three dimensional dimension chain equivalent exchanges into the information of two-dimensional plane dimension chain. And in the two-dimensional plane, the determination of the information of dimension chain, dimension loop and angle loop are important.

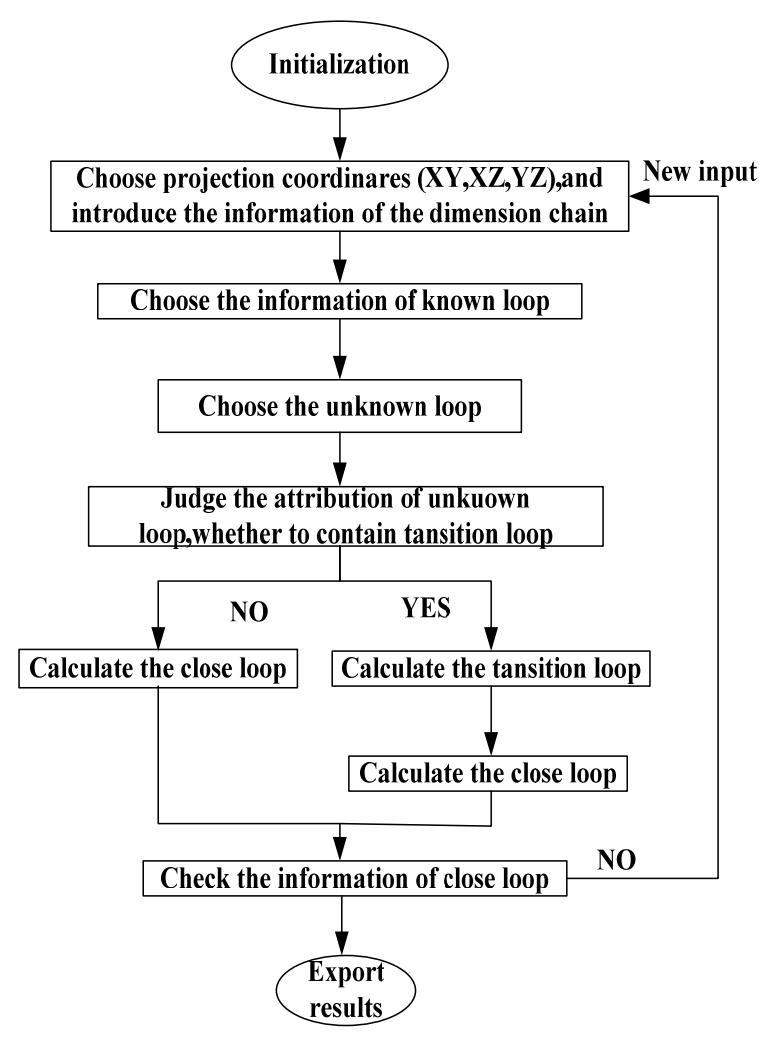

Fig.3 Flow chart 
According to the simplicity of VB.net software development, we chose VB.NET to realizing the secondary development of UG NX. And we designed the main operation interface of computer aided calculating plane dimension chain, which mainly including the choice button of the closed loop, the component loop and the transition loop, and picking up the known information of plane dimension chain, and calculational button of transitional loop and closed loop, and displaying dimension chain expression equation which is produced through the projection. The right part is the video conversion button, which is mainly used to change the visual angle of UG 3D interface. If we want to calculate dimension chain in one view, we must change view direction to convenient users find the information of the dimension chain, as shown in Fig.4, it is a part two-dimension plane figure which expresses informations of 3D dimension chain equivalent exchange into the information of the 2D dimensional chain.

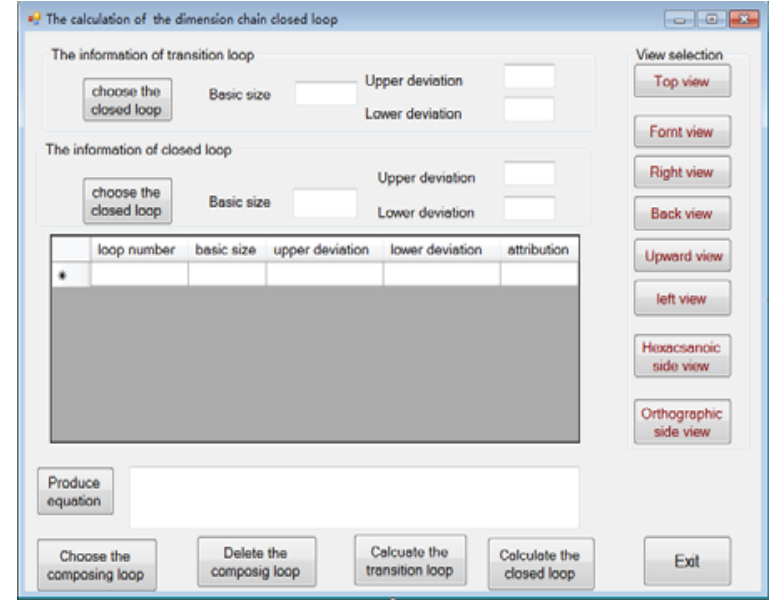

Fig.4 Operation interface

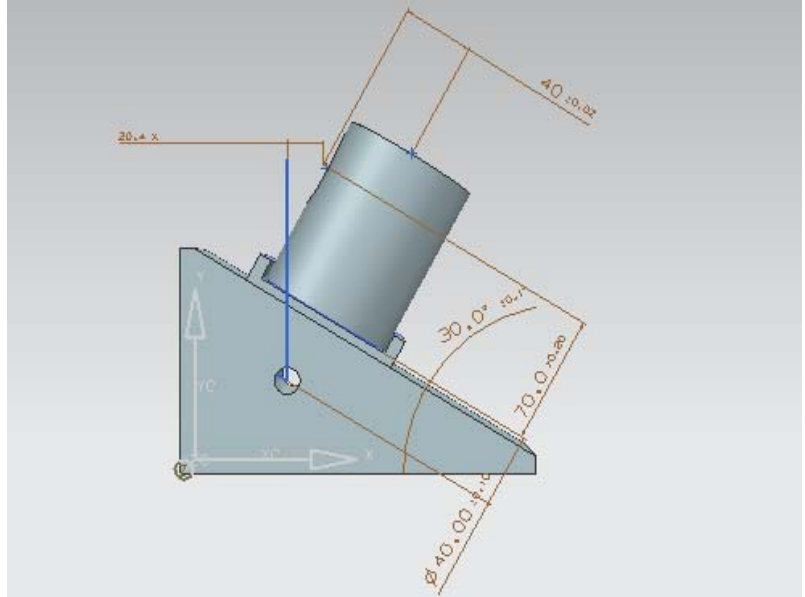

Fig.5 The dimension chain figure of 3D part

\section{Conclusion}

The projection method, which transforming two-dimensional plane dimension chain into one dimensional linear dimension chain, is applied in the current paper. And for the calculation of the angle of dimension chain, the vector angle computing method is used. It makes the process of 2D plane dimension chain calculation easier and clearer. It can judge increasing and decreasing of loop automatically. This method is convenient for computer program.

\section{References}

[1] X.M.Wang, S.Q.Liu: Ceramic Research and Vocational Education. Vol.6 (2008) No.1, p32-34

[2] J.L.Tang, J.S.Wang andX.L.Wei: Guangxi Journal of Light Industry. Vol.1 (2007), p98-80 (In Chinese)

[3] J.L.Tang, J.S.Wang andX.L.Wei: Modern Machinery. Vol.1 (2008), p32-34

[4] X.L.Wei: Computer-aided Tolerance Design in Plane Dimensional Chain. (University Southwest of Science and Technology, chain 2006).

[5] X.L.Wei, J.S.Wang: Engineering \& Automation, Vol. 38(2008), p72-73. (In Chinese) 